Classification

Physics Abstracts

$42.20-42.78-04.80$

\title{
Numerical model of transient thermal effects in high power optical resonators
}

\author{
Patrice Hello $\left({ }^{1,}{ }^{2}\right)$ and Jean-Yves Vinet $\left({ }^{1}\right)$ \\ (') Laboratoire d'Optique Appliquée, Ecole Polytechnique, ENSTA, Centre de l'Yvette, 91120 \\ Palaiseau, France \\ (2) Laboratoire de l'Accélérateur Linéaire, Groupe VIRGO, Bâtiment 208, Université d'Orsay, \\ 91405 Orsay, France
}

(Received 17 July 1992, accepted in final form 5 October 1992)

\begin{abstract}
We present a non-linear model of a Fabry-Perot resonator where the stored power can heat the mirrors and induce thermal aberrations, which, in turn, change the resonant wave. We first describe a sequential modelling of the temperature evolution, thermal aberrations and stored power; this model is then implemented with the help of a numerical propagation code. We give numerical examples in the field of cavities involved in gravitational wave interferometric antennas, for which thermal problems may arise and restrict their sensitivity.
\end{abstract}

\section{Introduction.}

In this paper, we present the modelling and the numerical simulation of a Fabry-Perot optical cavity where time dependent thermal effects can occur and affect the quality of the mirrors. These thermal effects are due to light power absorption in the mirrors which may induce temperature gradients, then a thermal lensing effect and thermoelastic distortions of the reflective surface and the refractive substrates. In turn, these thermal aberrations decrease the power stored in the cavity; the problem is thus nonlinear and it is more convenient to study it by numerical methods.

This work arises from the preliminary studies of the kilometric Fabry-Perot cavities involved in the projects of Interferometric Gravitational Wave Detectors, like the Italo-French VIRGO project or the American LIGO project [1] ; in the future operation of these Gravitational Wave antennas, there will be thousands of Watts stored in the cavities and hundreds of Watts crossing the input mirrors, making it necessary to investigate the thermal problems in the planned cavities. In other projects, like the German-British GEO project or the Australian AIGO project, delay lines are used instead of Fabry-Perot cavities and the thermal problem is not of the same kind.

More generally, thermal problems in cavities can occur each time high finesse cavities and high stored powers are considered: high power lasers, high accuracy measurements.. 
Actually, several authors have investigated the thermal effects, for example in laser systems, but in other contexts and with other approximations [2-6] that in our model which is original, because of the numerical treatment of the time dependancies and because of its accuracy.

The cavity we are going to study is (without loss of generality) a flat-spherical cavity (see Fig. 1), and we limit ourselves to the axi-symmetrical case for the input laser beam and for the optical system; the axial symmetry has the considerable advantage of allowing analytical solutions for the thermoelastic distortion of a mirror heated by a Gaussian beam [7-8]. The flat input mirror is a thick cylindrical block of pure silica with a coating on its cavity face; this mirror can thus absorb light power in the coating or in the bulk, with uniform absorption coefficients. The spherical end mirror, which is not crossed by light, may be manufactured from a material other than silica, in particular a material with a very small thermal expansion to thermal conductivity ratio (e.g. silicon or ULE), so we will neglect thermal effects in this mirror. The mirrors are supposed to be suspended by thin wires in a vacuum vessel with walls at temperature $T_{0}$. Heat losses are thus only due to thermal radiation, but we shall see in section 2 that the model is also relevant for convection losses, because we may linearize the radiative losses if the temperature increase due to light power absorption is not too strong [7].

We shall first describe the modelling of thermal effects in a cavity, with a sequential model for the temperature and thermally induced aberrations, and then we shall describe the numerical code we have built and the results we have obtained. We shall finally discuss the possibility of a general model where axial symmetry is no longer required, for example the case of a non-uniform absorption in the coating.

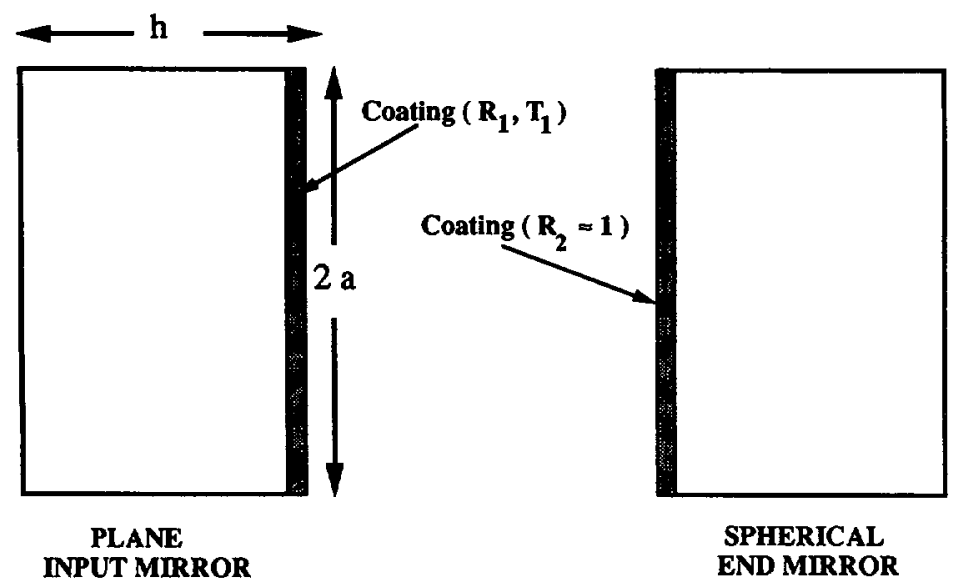

Fig. 1. - Sketch of the considered Fabry Perot resonator. $a$ and $h$ are the radius and the width of the input mirror, respectively.

\section{Modelling of thermal effects in a Fabry-Perot cavity.}

In previous papers [7, 8], we have derived time dependent analytical formulas for the temperature distribution, thermal lensing and thermal distortion of the coating for a thick plane mirror illuminated by a high power laser beam. We have expressed the temperature as a rapidly converging Fourier-Dini series like in [5] and in [9] as well as the induced thermal aberrations. The coefficients of these series depend linearly on the power distribution that reaches or crosses the mirror, and we can now develop a sequential model for the temperature and the thermal aberrations. 
2.1 SEQUENTIAL MODEL FOR THE TEMPERATURE OF THE INPUT MIRROR. - Within the assumption of axial symmetry (beams and mirrors), let us recall that we can generally express the time dependent temperature distribution of the cylindrical input mirror, of radius $a$ and thickness $h$, as a Fourier-Dini series, namely (see [7] for notation) :

$$
T(t, r, z)=\sum_{p, m}\left[S_{p m} \cos \left(u_{p} z / a\right)+A_{p m} \sin \left(v_{p} z / a\right)\right] J_{0}\left(k_{m} r\right)
$$

where $S_{p m}$ and $A_{p m}$ are (time dependent) real coefficients $u_{p}$ is the $p$-th solution of

$$
u=\tau \cot \left[\frac{u h}{2 a}\right]
$$

$v_{p}$ is the $p$-th solution of

$$
v=-\tau \tan \left[\frac{v h}{2 a}\right]
$$

and $a k_{m}$ is the $m$-th solution of :

$$
x J_{1}(x)-\tau J_{0}(x)=0
$$

where $\tau$ is the reduced radiation constant (if the heat losses are radiative) :

$$
\tau=\frac{4 \varepsilon \sigma T_{0}^{3} a}{K}
$$

( $\varepsilon$ is the emissivity of the silica, $\sigma$ is the Stefan-Boltzmann constant and $K$ is the thermal conductivity of the silica : $K=1.38 \mathrm{Wm}^{-1} \mathrm{~K}^{-1}$ ) or the reduced convective constant (if the heat losses are convective):

$$
\tau=\frac{\lambda_{\text {conv }} a}{K}
$$

( $\lambda_{\text {conv }}$ is the surface heat transfer coefficient).

A sequential modelling consists of cutting the time in successive slices of duration $\Delta t$, and in considering that all the heat sources have the same intensity during $\Delta t$. This requires that $\Delta t \ll t_{\mathrm{c}}$, if $t_{\mathrm{c}}$ is the evolution time of the temperature (time required to get the stationary state); in [7] we have found in the case of the large Virgo mirrors that $t_{c}$ is of the order of several hours, this is clearly not a very difficult condition to fill. For smaller mirrors $t_{c}$ will be much less, and $\Delta t$ has to be appropriately chosen.

Then at the time $t_{n}\left(t_{n}=t_{0}+n \Delta t\right)$, we may express the temperature distribution $T_{n}$ as :

$$
T_{n}(r, z)=\sum_{p, m}\left[S_{p m}^{(n)} \cos \left(u_{p} z / a\right)+A_{p m}^{(n)} \sin \left(v_{p} z / a\right)\right] J_{0}\left(k_{m} r\right)
$$

We know the temperature $T_{n}$ at time $t_{n}$, and the light powers that reach or cross the mirror, which are supposed to be constant between $t_{n}$ and $t_{n+1}$, so we can obtain the temperature at time $t>t_{n}$ from the resolution of the Fourier equation, the initial condition being :

$$
T(0, r, z)=T_{n}(r, z)
$$


so from [7] we get, if $t>t_{n}, T(t, r, z)$ as a steady-state solution [10] plus a transient solution :

$$
\begin{aligned}
T(t, r, z)=\sum_{m, p} & {\left[s_{p m}^{(n)} \cos \left(u_{p} z / a\right)+a_{p m}^{(n)} \sin \left(v_{p} z / a\right)\right] J_{0}\left(\zeta_{m} r / a\right)+} \\
& +\sum_{m, p}\left[u_{p m}^{(n)} \mathrm{e}^{-\alpha_{p m}\left(t-t_{n}\right)} \cos \left(u_{p} z / a\right)+v_{p m}^{(n)} \mathrm{e}^{-\beta_{p m}\left(t-t_{n}\right)} \sin \left(v_{p} z / a\right)\right] J_{0}\left(\zeta_{m} r / a\right)
\end{aligned}
$$

where the coefficients $s_{p m}^{(n)}, a_{p m}^{(n)}, u_{p m}^{(n)}$ et $v_{p m}^{(n)}$ can be determined for each heat source and will be explicited in the following, and :

$$
\alpha_{p m}=\frac{K}{\rho C a^{2}}\left[u_{p}^{2}+\zeta_{m}^{2}\right] \text { and } \beta_{p m}=\frac{K}{\rho C a^{2}}\left[v_{p}^{2}+\zeta_{m}^{2}\right]
$$

( $\rho$ is the density of the silica : $\rho=2202 \mathrm{~kg} \mathrm{~m}^{-3}, C$ its specific heat : $C=745 \mathrm{~J} \mathrm{~kg}^{-1} \mathrm{~K}^{-1}$, and $\zeta_{m}=a k_{m}$ ). The reverse of $\alpha_{p m}$ and $\beta_{p m}$ will be referred to as the time constants, the largest one being, $1 / \alpha_{00}$, in our numerical examples.

Then with the « initial conditions »:

$$
T_{n}(r, z)=T\left(t_{n}, r, z\right) \text { and } T_{n+1}(r, z)=T\left(t_{n+1}, r, z\right)
$$

we can express $T_{n+1}$ from $T_{n}$. Indeed, if we write $T_{n+1}$ as :

$$
T_{n+1}(r, z)=\sum_{p, m}\left[S_{p m}^{(n+1)} \cos \left(u_{p} z / a\right)+A_{p m}^{(n+1)} \sin \left(v_{p} z / a\right)\right] J_{0}\left(k_{m} r\right)
$$

we get from (9) and (11), and using $t_{n+1}-t_{n}=\Delta t$ :

$$
\left\{\begin{array}{l}
S_{p m}^{(n)}=s_{p m}^{(n)}+u_{p m}^{(n)} \\
A_{p m}^{(n)}=a_{p m}^{(n)}+v_{p m}^{(n)}
\end{array}\right.
$$

and

$$
\left\{\begin{array}{l}
S_{p m}^{(n+1)}=s_{p m}^{(n)}+u_{p m}^{(n)} \mathrm{e}^{-\alpha_{p m} \Delta t} \\
A_{p m}^{(n+1)}=a_{p m}^{(n)}+v_{p m}^{(n)} \mathrm{e}^{-\beta_{p m} \Delta t}
\end{array}\right.
$$

when eliminating $u_{p m}^{(n)}$ and $v_{p m}^{(n)}$ we get the recurrence relations :

$$
\left\{\begin{array}{l}
S_{p m}^{(n+1)}=\left(1-\mathrm{e}^{-\alpha_{p m} \Delta t}\right) s_{p m}^{(n)}+S_{p m}^{(n)} \mathrm{e}^{-\alpha_{p m} \Delta t} \\
A_{p m}^{(n+1)}=\left(1-\mathrm{e}^{-\beta_{p m} \Delta t}\right) a_{p m}^{(n)}+A_{p m}^{(n)} \mathrm{e}^{-\beta_{p m} \Delta t}
\end{array}\right.
$$

Finally, if we know the temperature distribution $T_{n}(r, z)$ at time $t_{n}$, we can derive the temperature distribution $T_{n+1}(r, z)$ at time $t_{n+1}=t_{n}+\Delta t$; all we have to do is to compute the coefficients $s_{p m}^{(n)}$ and $a_{p m}^{(n)}$ of the Fourier-Dini series of the steady state temperature corresponding to the mirror heated by a given beam of power $P_{n}$. We will compute these coefficients in later sections, but let us first derive sequential expressions for the thermal aberrations.

2.2 SEQUENTIAL MODEL FOR THE THERMAL ABERRATIONS. - We first address the aberrations due to the thermal lensing effect. When crossing the heated mirror, the light sees a phase shift simply related to the temperature distribution by [7] :

$$
\varphi(t, r)=\frac{2 \pi}{\lambda} \frac{\mathrm{d} n}{\mathrm{~d} T} \int_{-h / 2}^{h / 2} T(t, r, z) \mathrm{d} z
$$


where $\mathrm{d} n / \mathrm{d} T$ is the derivative of the index with respect to the temperature (also called thermal coefficient of index); for silica, we have in vacuum and at $1.06 \mu \mathrm{m}: \mathrm{d} n / \mathrm{d} T=$ $-0.87 \times 10^{-5} \mathrm{~K}^{-1}$ If we consider the sequential model for temperature, the phase shift $\varphi_{n}(r)$ at the time $t_{n}$ is :

$$
\varphi_{n}(r)=\frac{2 \pi}{\lambda} \frac{\mathrm{d} n}{\mathrm{~d} T} \int_{-h / 2}^{h / 2} T_{n}(r, z) \mathrm{d} z
$$

Thus, with the expression (7) for $T_{n}$, we obtain :

$$
\varphi_{n}(r)=\frac{4 \pi a}{\lambda} \frac{\mathrm{d} n}{\mathrm{~d} T} \sum_{p, m} S_{\rho m}^{(n)} \frac{\sin \left(u_{p} h / 2 a\right)}{u_{p}} J_{0}\left(\zeta_{m} r / a\right) .
$$

So, we are able to compute at every time $t_{n}$ the aberrations due to the thermal lensing effect.

The aberrations due to the thermoelastic distortion of the coating can also be obtained by computing the phase shift seen by the light $\varphi(t, r)$ for a refraction; this phase shift is related to the coefficients $S_{p m}$ and $A_{p m}$ of the Fourier-Dini expansion of the temperature [8]:

$$
\begin{aligned}
& \varphi(t, r)=-\frac{2 \pi a}{\lambda} \frac{\nu}{\lambda+\mu} \times \\
& \quad \times \sum_{p, m}\left[\frac{\sinh \left(\gamma_{m}\right) P_{p m}}{\sinh \left(\gamma_{m}\right) \cosh \left(\gamma_{m}\right)+\gamma_{m}} S_{p m}+\frac{\cosh \left(\gamma_{m}\right) I_{p m}}{\sinh \left(\gamma_{m}\right) \cosh \left(\gamma_{m}\right)-\gamma_{m}} A_{p m}\right] J_{0}\left(\zeta_{m} \text { rla }\right)
\end{aligned}
$$

where $\nu$ is the stress temperature modulus, $\lambda$ is the first Lamé coefficient, $\mu$ is the second Lamé coefficient (we have for silica: $\nu=5.91 \times 10^{4} \mathrm{~J} \mathrm{~m}^{-3} \mathrm{~K}^{-1}, \lambda=1.56 \times 10^{10} \mathrm{~J} \mathrm{~m}^{-3}$, $\left.\mu=3.13 \times 10^{10} \mathrm{~J} \mathrm{~m}^{-3}\right)$, and :

$$
\begin{aligned}
& P_{p m}=\frac{u_{p} \cosh \left(\gamma_{m}\right) \sin \left(\xi_{p}\right)+\zeta_{m} \sinh \left(\gamma_{m}\right) \cos \left(\xi_{p}\right)}{u_{p}^{2}+\zeta_{m}^{2}} \\
& I_{p m}=\frac{v_{p} \sinh \left(\gamma_{m}\right) \cos \left(\eta_{p}\right)-\zeta_{m} \cosh \left(\gamma_{m}\right) \sin \left(\eta_{p}\right)}{v_{p}^{2}+\zeta_{m}^{2}}
\end{aligned}
$$

(with : $\xi_{p}=u_{p} h / 2 a, \eta_{p}=v_{p} h / 2 a, \gamma_{m}=\zeta_{m} h / 2 a$ ).

At time $t_{n}$, the temperature is given by (7) and the phase shift is :

$$
\begin{aligned}
& \varphi_{n}(r)=-\frac{2 \pi a}{\lambda} \frac{\nu}{\lambda+\mu} \times \\
& \quad \times \sum_{p, m}\left[\frac{\sinh \left(\gamma_{m}\right) P_{p m}}{\sinh \left(\gamma_{m}\right) \cosh \left(\gamma_{m}\right)+\gamma_{m}} S_{p m}^{(n)}+\frac{\cosh \left(\gamma_{m}\right) I_{p m}}{\sinh \left(\gamma_{m}\right) \cosh \left(\gamma_{m}\right)-\gamma_{m}} A_{p m}^{(n)}\right] J_{0}\left(\zeta_{m} r / a\right) .
\end{aligned}
$$

Thus, we are able to compute at every time $t_{n}$ the aberrations due to the thermoelastic distortion of the coating of the mirror. Now we may find the aberrations induced by the different sources of heating.

2.3 HEAT SOURCES AND RELATED ABERRATIONS. - The input mirror can be heated by different sources (see Fig. 2) which may be treated separately, owing to the linear approximation used in the thermal equations.

2.3.1 Aberrations due to a coating dissipation of light power. - The coating can absorb power from two beams : the intra-cavity resonant wave and the (roughly Gaussian) incoming 


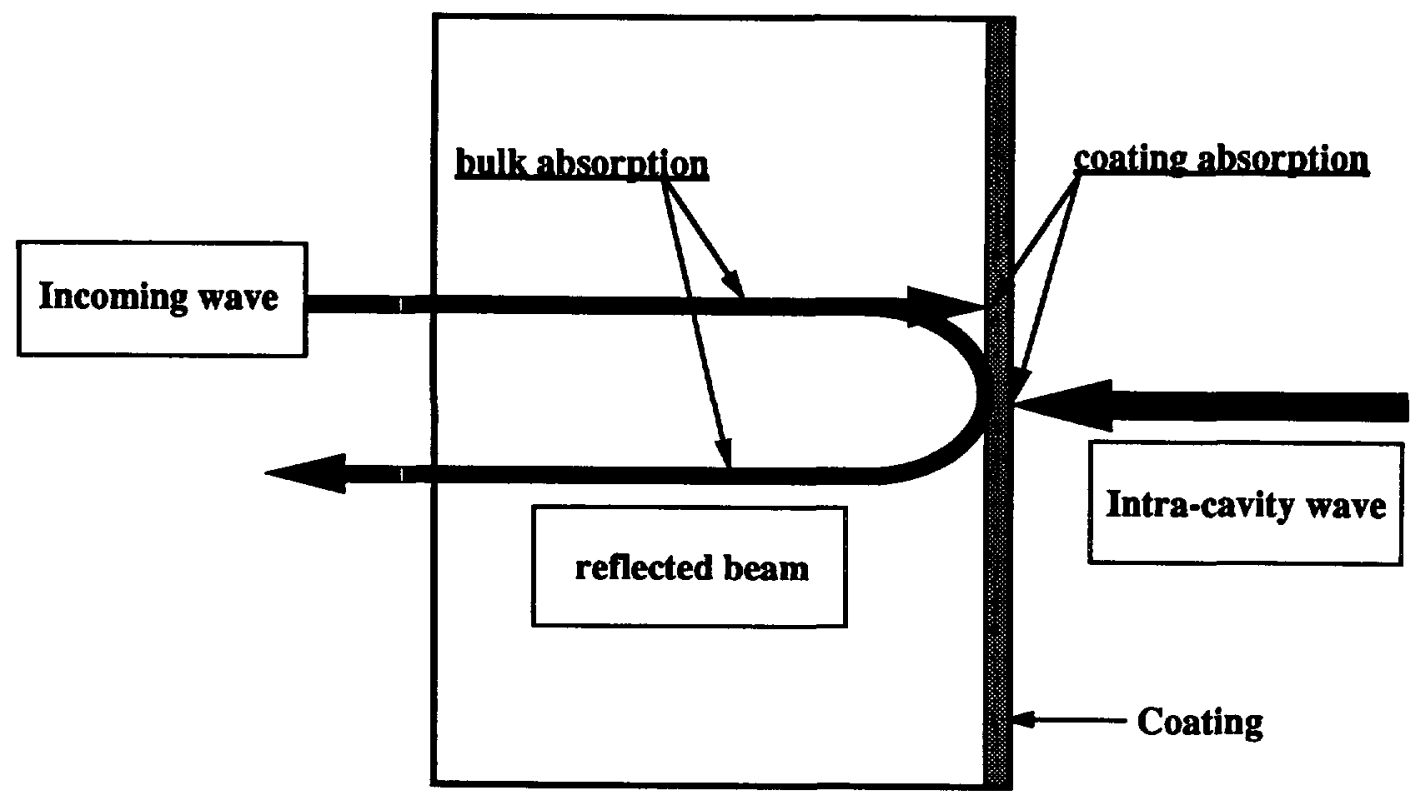

Fig. 2. - Heat sources for the input mirror of the cavity. The mirror can be heated in its bulk by the two beams which cross it, and by coating absorption of the resonant intra-cavity wave and of the incoming wave.

wave. We may neglect the contribution of the second one, because of the power resonance in the cavity. The thermal aberrations are then given by (18) and (22) and directly related to the Fourier-Dini expansion of the temperature; according to the sequential model for the temperature (9), we need the steady state coefficients $s_{p m}^{(n)}$ and $a_{p m}^{(n)}$ in order to compute the temperature and then the aberrations at each time $t_{n}$. We get these coefficients from [7] :

$$
\begin{aligned}
& s_{p m}^{(n)}=\frac{2 \varepsilon a^{2}}{K h} p_{m}^{(n)} \frac{\cos \left(u_{p} h / 2 a\right)}{\left(\zeta_{m}^{2}+u_{p}^{2}\right) c_{p}} \\
& a_{p m}^{(n)}=-\frac{2 \varepsilon a^{2}}{K h} p_{m}^{(n)} \frac{\sin \left(v_{p} h / 2 a\right)}{\left(\zeta_{m}^{2}+v_{p}^{2}\right) s_{p}}
\end{aligned}
$$

where $\varepsilon$ is the power dissipation coefficient of the coating, $c_{p}$ and $s_{p}$ are given by:

$$
c_{p}=1+\frac{a}{u_{p} h} \sin \left(u_{p} h / a\right) \text { and } s_{p}=1-\frac{a}{v_{p} h} \sin \left(v_{p} h / a\right)
$$

and the $p_{m}^{(n)}$ are the coefficients of the Dini expansion of the power distribution $P^{(n)}$ that reaches the coating at time $t_{n}$.

$$
P^{(n)}(r)=\sum_{m} p_{m}^{(n)} J_{0}\left(k_{m} r(a)\right.
$$

and then :

$$
p_{m}^{(n)}=\frac{2 \zeta_{m}^{2}}{\zeta_{m}^{2}+\tau^{2}} \frac{1}{J_{0}\left(\zeta_{m}\right)^{2}} \frac{1}{a^{2}} \int_{0}^{a} P^{(n)}(r) J_{0}\left(\zeta_{m} r / a\right) r \mathrm{~d} r
$$


2.3.2 Aberrations due to the bulk absorption of light power. - In figure 2 we see that two beams of roughly the same intensity can heat the bulk of the mirror, namely those which cross the mirror : the incoming beam and the beam reflected by the cavity. In order to derive the thermal aberrations induced by the bulk absorption of these two beams, we need the steadystate coefficients $s_{p m}^{(n)}$ and $a_{p m}^{(n)}$ which are given by [7] :

$$
\begin{aligned}
s_{p m}^{(n)} & =\frac{4 \alpha a^{3}}{K h} p_{m}^{(n)} \frac{1}{\zeta_{m}^{2} c_{p}}\left[\frac{\sin \left(u_{p} h / 2 a\right)}{u_{p}}-\tau \frac{\cos \left(u_{p} h / 2 a\right)}{\zeta_{m}^{2}+u_{p}^{2}}\right] \\
a_{p m}^{(n)} & =0
\end{aligned}
$$

where $\alpha$ is the lineic absorption coefficient of the material.

Note that, in this case, if at the initial time $t_{0}$ the mirror is at thermal equilibrium with the external vessel, then the odd coefficients $A_{p m}^{(n)}$ always vanish, because of the recurrence relation (15) and because $a_{p m}^{(n)}=0$.

Moreover, the coefficients $p_{m}^{(n)}$ come from two contributions :

$$
p_{m}^{(n)}=\sigma_{m}^{(n)}+\pi_{m}^{(n)}
$$

The first term corresponds to the incoming beam ; we have for example, if the latter a pure TEM $_{00}$ Gaussian mode, perfectly mode matched to the cavity, with a constant power $P$, and a waist $w$ :

$$
\varpi_{m}^{(n)}=\frac{P}{\pi a^{2}} \frac{\zeta_{m}^{2}}{\left(\zeta_{m}^{2}+\tau^{2}\right) J_{0}\left(\zeta_{m}\right)^{2}} \exp \left(-\frac{1}{8} \zeta_{m}^{2} \frac{w^{2}}{a^{2}}\right)
$$

The second term corresponds to the reflected beam of unknown geometry and has to be computed at each time $t_{n}$, using (27).

Finally, using the recurrence relations (15), the above formulas for the steady state coefficients $s_{p m}^{(n)}$ and $a_{p m}^{(n)}$ and for coefficients of the Dini expansion of the power distribution, we are able to express, at each time $t_{n}$, the thermal aberrations induced by absorption of light power either in the bulk or in the coating of the input mirror of the considered cavity. In fact, what is changing between two sequences (between the time $\Delta t$ ) is the power distribution that crosses or hits the mirror, and the aberrations are perfectly determined from this distribution. So, we can use this theoretical work in a dynamical numerical code of a Fabry-Perot cavity, where there is a coupling between the computation of the thermal aberrations and the optical computation.

\section{Simulation of a Fabry-Perot illuminated by an intense laser beam.}

It is clear that a non-linear coupling occurs between the thermal system which determines the optical tuning, and the optical system which determines the rate of heating. We have thus to build a model of a cavity, considering the action of the wave front thermal distortion of the stored power and the feedback of the stored power upon the heating rate. This is possible by mixing the previous sequential model for thermal aberrations and a numerical code computing stored waves and reflected waves in a Fabry-Perot cavity.

\subsection{DESCRIPTION OF THE NUMERICAL CODE.}

3.1.1 Optical simulation. - The numerical code is based upon a static purely optical code which is described elsewhere [11-13]. In a few words, all the transformations undergone by the 
light wave in the cavity are represented by linear operators acting on the amplitude of the lightwave. This method allows us to consider a large range of defects of the mirrors : finite size, curvature errors, aberrations, index inhomogeneities, tilts and misalignments.

For example, the propagation between the two mirrors in the cavity may be written as the convolution product of the wave amplitude by a paraxial Green function $G$, which is, in the Fourier Space :

$$
G(p, q)=\mathrm{e}^{i k L} \mathrm{e}^{-i \frac{\lambda L}{4 \pi}\left(p^{2}+q^{2}\right)}
$$

$L$ being the propagation length and $\lambda$ the wavelength of light.

The linear propagation operator may be thus expressed as :

$$
\mathfrak{S}=\mathfrak{F}^{-1} G \mathcal{F} \text {. }
$$

where $\mathfrak{F}$ is the direct Fourier transform. This allows us to use FFT routines like in other similar methods of beam propagation [14].

The refraction and reflection operators associated with a mirror having scalar amplitude transmission and reflection coefficients $t$ and $r$, are more simply, assuming a $\pi / 2$ phase change for a reflection :

$$
\mathfrak{J}(x, y)=t \mathrm{e}^{l f(x, y)} d(x, y) \text { and } \mathcal{R}(x, y)=i r \mathrm{e}^{\ell \theta(x, y)} d(x, y)
$$

where $d(x, y)$ is an aperture function, $f(x, y)$ and $g(x, y)$ represent the local phase change due to either the reflecting surface shape or the variable optical thickness. These functions are fully explained in [13].

Now, if we consider a whole Fabry-Perot cavity (see Fig. 3 for notation), the continuity equations verified by the waves on the mirrors lead to the implicit equation for the amplitude of the intra-cavity wave $\Psi_{1}$.

$$
\Psi_{1}=\mathfrak{J}_{1} \Psi_{\text {In }}+\mathcal{R}_{1} \mathfrak{P} \mathcal{R}_{2} \mathfrak{T} \Psi_{1}
$$

\section{INPUT MIRROR}

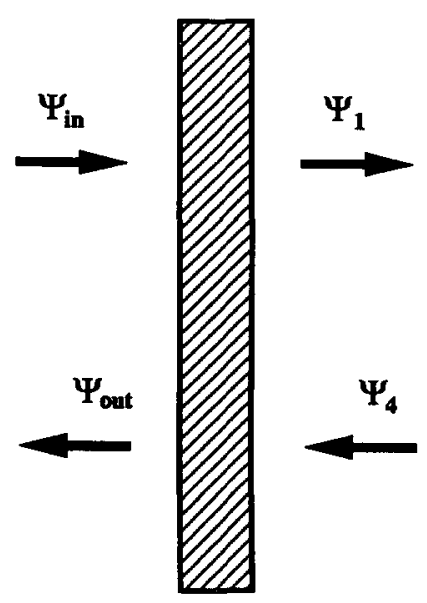

END MIRROR

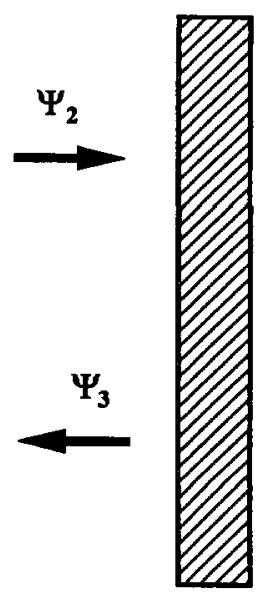

Fig. 3. - Waves in the cavity (notation). 
The simulation of a cavity is based upon the resolution of this equation; we solve it by successive iterations from a first approximate solution that we may choose equal to the exact eigenmode of the ideal cavity, until the required accuracy is reached.

An important point of our model is that we are able to tune very simply the cavity at resonance, whatever the defects of the mirrors, provided they are small, especially if we consider the thermal aberrations. Indeed a perturbation calculation concludes [13], to first order, that the cavity is resonant with the Gaussian TEM $_{00}$ mode if we add to the phase of the propagation operator the global phase $-\operatorname{Arg}(\lambda)$, where $\lambda$ is the Hermitian scalar product :

$$
\lambda=\left\langle\mathcal{R}_{1} \mathfrak{T} \mathcal{R}_{2} \mathfrak{T} \Psi_{00} \mid \Psi_{00}\right\rangle
$$

and $\Psi_{00}$ is the purely Gaussian TEM $_{00}$ mode of the unperturbed cavity. This method, very useful in order to servo the tuning of the cavity, requires the simulation of only one round trip in the cavity and is therefore unexpensive in computer time.

3.1.2 Simulation of the thermo-optical coupling. - The simulation involves two parts : an optical simulation, described in the previous section, and a computation of the thermal aberrations, described in section 2 . The simplest code would consist in computing the intracavity wave at each round trip of the light in the cavity, and to derive the thermal aberrations related to the power distributions reaching the coating or crossing the mirror but the thermal effects are very slow to develop. For instance, if we compute the largest time constant $t_{0}$.

$$
t_{0}=\frac{1}{\alpha_{00}}=\frac{\rho C a^{2}}{K} \frac{1}{u_{0}^{2}+\zeta_{0}^{2}}
$$

we note that $t_{0}$ is several hours in our examples, and we thus would have to follow the evolution of the cavity during a time of the same magnitude ; this would require some billions of iterations. In fact, because of the large value of $t_{0}$, the evolution of the thermal aberrations is quasi-static with respect to the optical time constants, such as the storage time $\tau_{\mathrm{s}}$ or the decay time. This important remark means that during the storage time of the cavity the thermal aberrations remain almost unchanged, and allows the sequential numerical modelling shown in table I.

This method is of course valid if we choose $\Delta t$ larger than the storage time $\tau_{\mathrm{s}}$ of the cavity :

$$
\Delta t>\tau_{\mathrm{s}} .
$$

But, in this form, the method is yet too expensive in computer time, and we must find a way to accelerate the simulation. Fortunately, we may squeeze the time scale; indeed, the quantities that appear in the time constants are the radius a of the mirror, the thermal conductivity $K$, the density $\rho$ and the specific heat $C$. The first, two $a$ and $K$, occur in the expressions of the amplitude of the thermal aberrations, but $\rho$ and $C$ occur only in the time constants. We may choose these last two parameters as we want in order to decrease $t_{0}$ and all the other time constants : we shall not change the amplitude of the thermal effects, only their evolution time scale. Thus we can squeeze the time scale by decreasing the time constants, but we have to keep the quasi-static evolution property of the thermal system, namely: $t_{0} \gg \tau_{\mathrm{s}}$.

It is also useful to keep $\Delta t \ll t_{0}$ in order to obtain a good accuracy in the time evolution. Note that this quasi-static approximation does not allow us to study accurately phenomena with a short time scale ( short » means very small with respect to $t_{0}$ ), such as possible short period oscillations (the code would see only the envelope of these oscillations), or thermally induced bistability. In principle, it would be possible to look at such phenomena, but, practically, the quasi-static approximation is needed because of prohibitive computer time. 
Table I. - Briefly summarized structure of the loop-over-time of the numerical code.

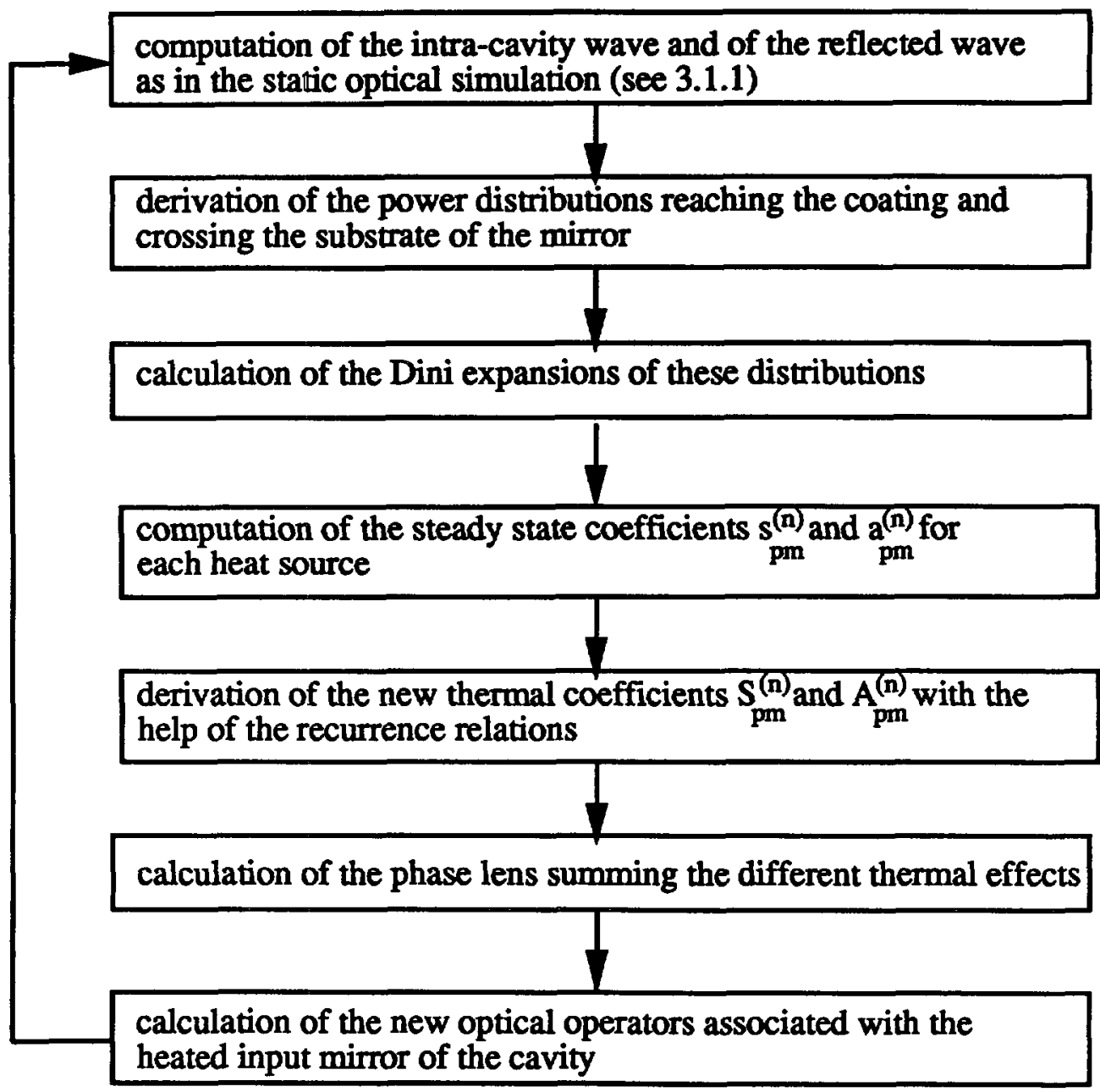

In the following simulations, we have chosen a squeezed time corresponding to the time parameter : $\rho C a^{2} / K=1 \mathrm{~s}$, and an iteration time $\Delta t=1 \mathrm{~ms}$. As we consider a cavity with a storage time $\tau_{\mathrm{s}}$ about $0.1 \mathrm{~ms}$, all the previous conditions are fullfilled, and the steady state is obtained after only a few hundred iterations. Now, we can give some numerical results about the behaviour of a Fabry-Perot cavity illuminated by an intense laser beam.

3.2 NUMERICAL RESULTS. - The code has been implemented with the FORTRAN language on the Siemens VP200 vector machine at the CIRCE center (Orsay, France), and has been linked with the vectorized SSL-II library, for use of the 2-D complex FFT and of the Bessel functions; a vector machine is indeed suitable, because the optical part of the code can be highly vectorized $[12,13]$ (the optical operators as well as the light waves are represented by 2$\mathrm{D}$ arrays, and the action of an operator upon a wave is simply represented by a multiplication between two arrays).

The examples shown in the following sections are chosen in the context of the optical design of the Virgo cavities. These are $3 \mathrm{~km}$ long cavities, with a flat input mirror and a spherical end mirror of $3.45 \mathrm{~km}$ curvature radius. The mirrors have a radius of $30 \mathrm{~cm}$ and a width of $20 \mathrm{~cm}$; 
the power reflection coefficients of the mirrors are 0.85 for the input mirror and near unity for the end mirror, the finesse is thus about 40 . The walls of the vacuum tanks are supposed to be at the constant temperature $T_{0}=300 \mathrm{~K}$.

Note that, with such mirrors, numerically tested convergence and accuracy criteria allow us to truncate the Fourier-Dini expansions for thermal aberrations at about the 50-th order, for which the double precision (15 digits) is reached for the computation of the temperature as well as of the thermal aberrations; the speed of the numerical code is thus mainly limited by the optical part and particularly by the many double precision complex 2D-FTT's we have to compute.

3.2.1 Cavity with a fixed tuning. - We may first simulate the behaviour of a free cavity, i.e. without servo; the cavity is first tuned on its fundamental mode, with the help of the fast subroutine described in section 3.1.1 (Eq. (36)), and then illuminated by an ideal $\mathrm{TEM}_{00}$ Gaussian beam, which is perfectly adapted to the ideal cavity.

A numerical result is shown in figure 4 ; the incoming power is equal to $250 \mathrm{~W}$ and the coating absorption coefficient is $10^{-4}$, which is a correct order of magnitude for current technology. Note that we have neglected the bulk absorption, since it is weak compared to the coating absorption. We see a straight decrease of the power stored in the cavity, which stabilizes after about $40 \mathrm{mn}$ of operation.

The power falls mainly because the cavity goes out of resonance, due to the thermal distorsions of the heated input mirror, but we can servo the tuning of the cavity.

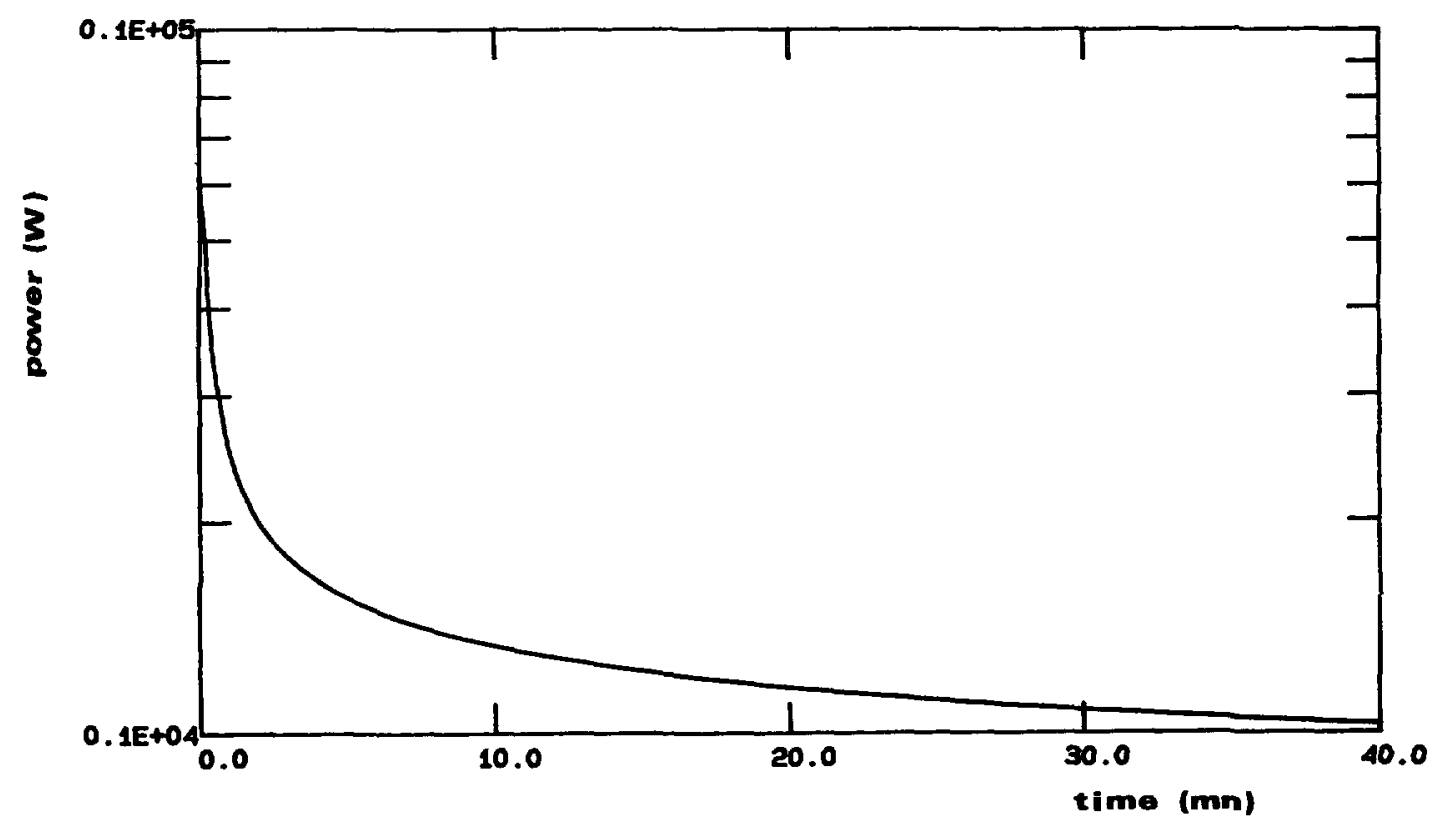

Fig. 4. - Evolution of the stored power when the cavity is tuned at resonance at the initial time. The incoming power is $250 \mathrm{~W}$ and the coating absorption is $10^{-4}$

3.2.2 Cavity with a servoed tuning. - The servos have response times much shorter than the thermal characteristic times and even than the storage time of the cavity, so that we may consider that the cavity is at resonance at each time. Thus, we simulate a servoed tuning by 
adjusting, at each occurrence of the optical code, the resonance of the cavity, with a subroutine based upon the method outlined in section 3.1.1 (see Eq. (36)).

In figure 5 we present a numerical result obtained with an incoming power of $250 \mathrm{~W}$ and coating absorption coefficients of $10^{-4}$ and $10^{-5}$. In the first case, there is a decrease of power but less than in the example of the previous section (there is a factor between initial intracavity power and steady-state stored power instead of a factor 5). This reduction is now only due to a decreasing coupling between the incoming pure Gaussian wave and the resonant mode of the cavity (note that more than two hours are needed to reach the steady state). In the case of a $10^{-5}$ absorption, the decrease of power appears negligible. We also give in this case the

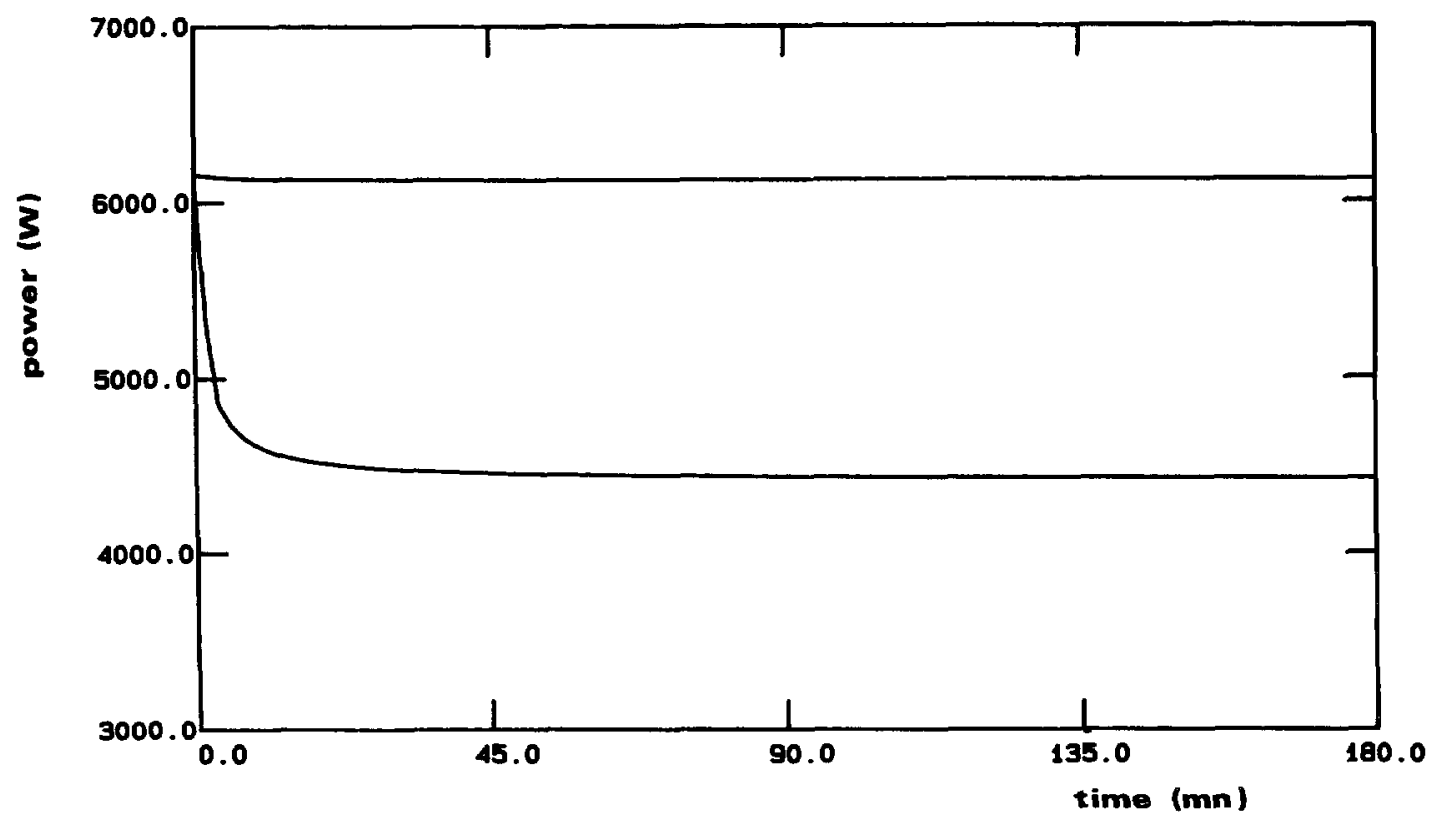

Fig. 5. - Evolution of the stored power with a servoed tuning of the resonance of the cavity. The incoming power is $250 \mathrm{~W}$ and the coating absorption is $10^{-5}$ (upper curve) or $10^{-4}$ (lower curve).

picture (Fig. 6) of the evolution of the temperature (modulo $T_{0}$ ) of the « hot point » of the mirror, namely the center of the coating, using expression (7); finally, figure 7 shows the evolution of the sagitta of the mirror, computed as the maximum of relative displacement on the mirror surface.

An important consequence of these numerical examples is that Interferometric Gravitational Wave Detectors like LIGO or VIRGO could be found to work well, if coatings with intrinsic absorption less than $10^{-5}$ could be made over large surfaces. This seems to be now feasible, if we can extend the best coating technology up to tens of centimeters. For instance, total losses (absorption and scattering) of about $10^{-6}$ have been recently measured on small coated mirrors [15]. 


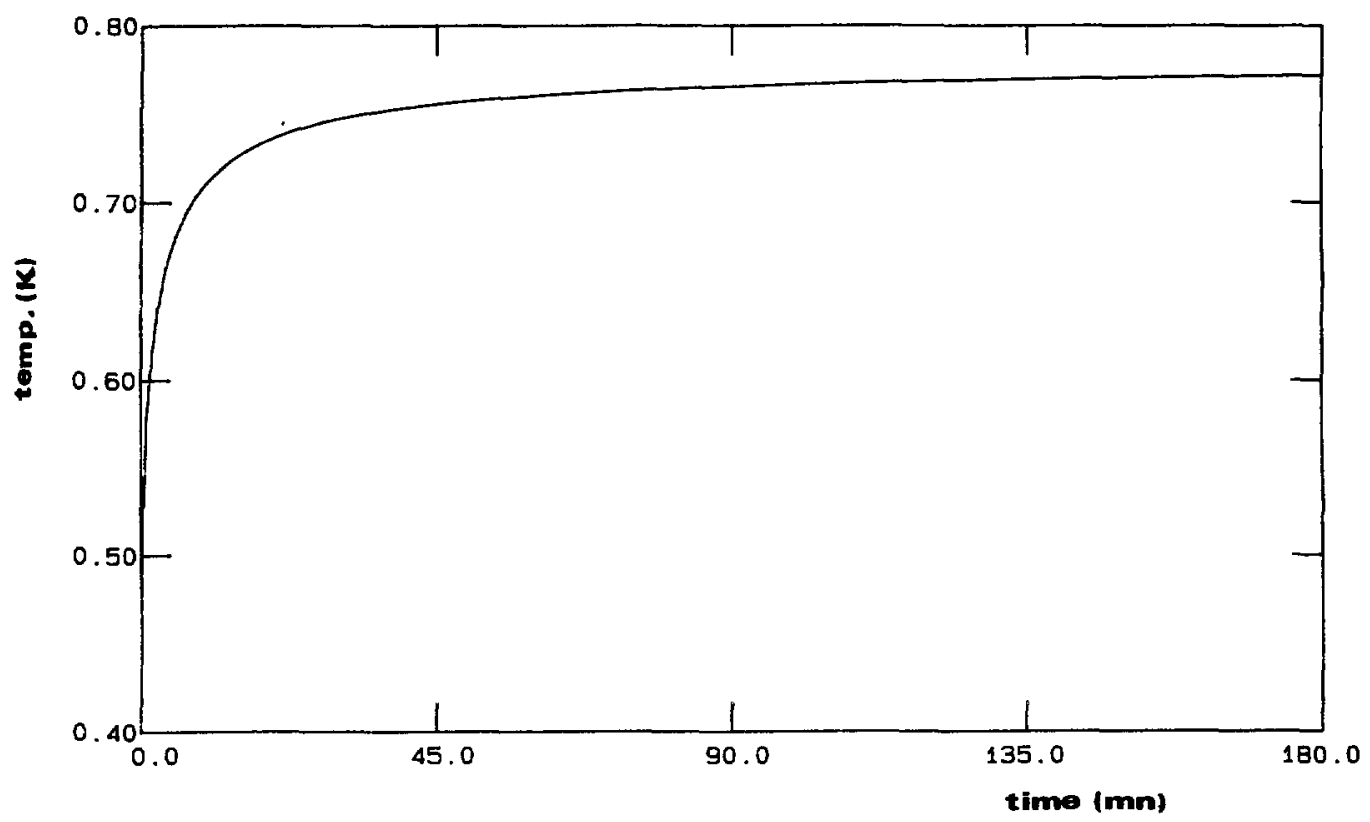

Fig. 6. - Evolution of the temperature of the « hot point » of the mirror, in the case of a servoed tuning of the cavity, a $250 \mathrm{~W}$ incoming power and $10^{-5}$ coating absorption.

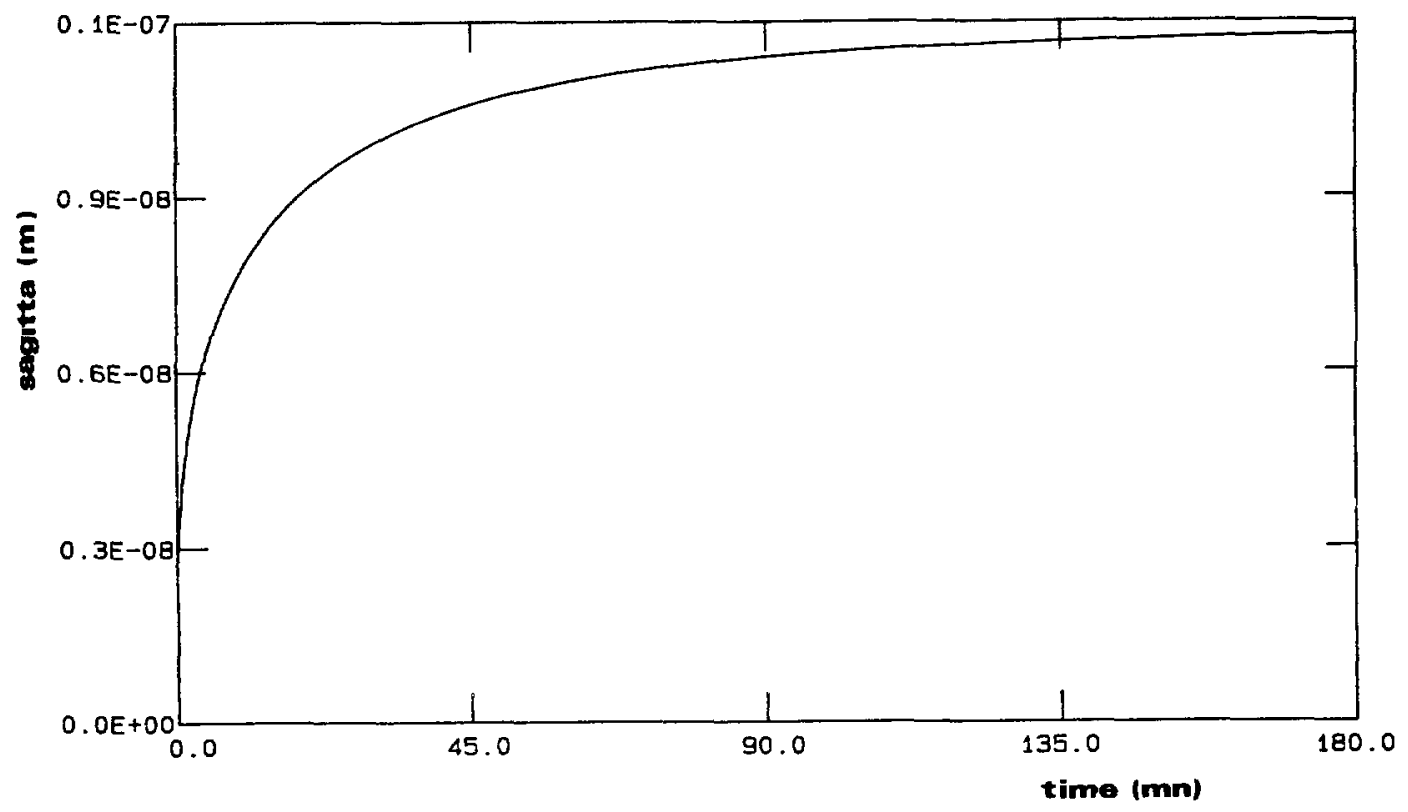

Fig. 7. - Sagitta of the heated mirror as a function of time, in the case of a servoed tuning of the cavity, a $250 \mathrm{~W}$ incoming power and $10^{-5}$ coating absorption. 


\section{Possibility of a numerical simulation without symmetry hypothesis.}

In this section we want to discuss the possibility of a more general code where we no longer restrict ourselves to the axially symmetrical case. In this symmetrical case, we have found analytical solutions for the temperature distribution, thermal lensing and thermoelastic distortion of a cylindrical mirror heated by a laser beam. When axial symmetry no longer applies, for instance when the absorbed power distribution is no longer a purely radial function, we have to express the temperature more generally :

$$
T(t, r, \theta, z)=\sum_{m, n, p}\left(A_{m n p} \cos \left(u_{n p} z\right)+B_{m n p} \sin \left(v_{n p} z\right)\right) \mathrm{e}^{\imath n \theta} J_{n}\left(k_{m n} r\right) .
$$

We shall refer to this expression as a generalized Fourier-Dini series.

As an example, it is straightforward to verify that the steady-state temperature distribution of a cylindrical mirror heated by a laser beam and with a non-uniform coating absorption $\varepsilon(r, \theta)$ or (equivalently) a non-radial power distribution $P(r, \theta)$ is :

$T(r, \theta, z)=\sum_{m, n} \frac{a p_{m n} \mathrm{e}^{-\zeta_{m n} h / 2 a}}{K} \frac{\left(\zeta_{m n}-\tau\right) \mathrm{e}^{-\zeta_{m n}(h-z) a}+\left(\zeta_{m n}+\tau\right) \mathrm{e}^{-\zeta_{m n} z / a}}{\left(\zeta_{m n}+\tau\right)^{2}-\left(\zeta_{m n}-\tau\right)^{2} \mathrm{e}^{-2 \zeta_{m n} h / a}} \mathrm{e}^{l n \theta} J_{n}\left(\zeta_{m n} r / a\right)$

with the same notation as in the previous sections, and where the coefficient $\zeta_{m n}$ is the $m$-th zero of :

$$
x J_{n-1}(x)+(\tau-n) J_{n}(x)
$$

and the $p_{m n}$ are the coefficients of the generalized Fourier-Dini expansion of the absorbed power distribution :

$$
p_{m n}=\frac{2 \zeta_{m n}^{2}}{\zeta_{m n}^{2}+\tau^{2}-n^{2}} \frac{1}{J_{n}\left(\zeta_{m n}\right)^{2}} \frac{1}{2 \pi a^{2}} \int_{0}^{2 \pi} \int_{0}^{a} \varepsilon(r, \theta) P(r, \theta) \mathrm{e}^{-\imath n \theta} J_{n}\left(\zeta_{m n} r / a\right) r \mathrm{~d} r \mathrm{~d} \theta
$$

if we use the normalization property of our particular set of Bessel functions [16].

Hence, following the method described in [7], expanding the hyperbolic functions of $z$ appearing in (40) as Fourier series, we can derive the general time-dependent temperature ab initio :

$$
\begin{aligned}
T(t, r, \theta, z)=\frac{2 a^{2}}{K h} & \sum_{m, n, p} p_{m n}\left[1-\mathrm{e}^{-\alpha_{m n p} t}\right] \frac{\cos \left(u_{p} h / 2 a\right)}{\left(\zeta_{m n}^{2}+u_{p}^{2}\right) c_{p}} \cos \left(u_{p} z / a\right) \mathrm{e}^{i n \theta} J_{n}\left(\zeta_{m n} r / a\right)- \\
& -\frac{2 a^{2}}{K h} \sum_{m, n, p} p_{m n}\left[1-\mathrm{e}^{-\beta_{m n p} t}\right] \frac{\sin \left(v_{p} h / 2 a\right)}{\left(\zeta_{m n}^{2}+v_{p}^{2}\right) s_{p}} \sin \left(v_{p} z / a\right) \mathrm{e}^{i n \theta} J_{n}\left(\zeta_{m n} r / a\right)
\end{aligned}
$$

where $u_{p}, v_{p}, c_{p}$ and $s_{p}$ have the same definition as in section 2 and:

$$
\begin{aligned}
& \alpha_{m n p}=\frac{K}{\rho C a^{2}}\left[\zeta_{m n}^{2}+u_{p}^{2}\right] \\
& \beta_{m n p}=\frac{K}{\rho C a^{2}}\left[\zeta_{m n}^{2}+v_{p}^{2}\right] .
\end{aligned}
$$


A similar expression can be obtained in the same way for inhomogenous power absorption in the substrate of the mirror. From these expressions for the temperature, and by analogy with equation (16), we may find the thermal lens profile, i.e. the total optical path distortion, after a single pass through the mirror, due to thermal lensing in the substrate of the mirror :

$$
\delta(t, r, \theta)=\frac{\mathrm{d} n}{\mathrm{~d} T} \int_{-h / 2}^{h / 2} T(t, r, \theta, z) \mathrm{d} z
$$

which can be explicitly calculated.

Therefore, we have shown that, in the general case of non-uniform absorption of the light power, we can derive analytical formulas for the temperature distribution and for the aberrations induced by the thermal lensing effect. As in section 2, we are also able to derive a sequential model for the temperature distribution and for the thermal lensing aberrations and find recurrence relations similar to equations (15).

But addressing the transient thermoelastic problem is another story. To our knowledge of the literature in this field of Physics, only Cutolo et al. [9] have considered this 3-D case for heated mirrors and computed the general solution for the steady-state temperature, but they restricted their study to axial symmetry (and steady-state) when they tackled the thermoelastic problem. The point is that there is no general analytical solution to the 3-D elastic problem, even for a simple solid like a cylindrical mirror (except if it can be approximated by a 2-D model like a plate $(h \ll a)$, or like a rod $(h \gg a)$, but this is not our case, and generally not the case for cavity or laser mirrors). The best we can do is to show that solutions exist [17]. An alternative numerical solution would be, for example, to add to the code a routine computing the thermoelastic deformation of the mirror surface by a finite-element method ; this routine would take the data given by the analytical calculation of the temperature field, evaluate the local temperature gradients and finally derive the shape of the reflecting face of the mirror.

In principle, such a code should work, but it would be much slower, firstly because of the computation of the generalized Fourier-Dini series for the temperature and the thermal lens expressions (the computation requires one more loop, corresponding to the angular Fourier expansion), and secondly because of the computation of the thermoelastic distortion of the mirror by a finite-element method. But this code could test the thermal behavior of cavities or lasers with more complex geometries, when the mirrors are tilted with respect to the beam axis, as in ring resonators for example. We are going to develop such a numerical code and test it.

\section{Conclusion.}

In this paper, we describe a non-linear model of Fabry-Perot cavities where thermal effects induced by the stored power are taken into account. Some numerical results have been obtained in the cases of fixed tuning as well as servoed tuning. Some ideas concerning the realisation of a more general numerical code have also been given in section 4 , where we propose the use of a finite-element method in order to compute the thermoelastic distortion of the mirror.

The results are important in the field of gravitational wave interferometric detection, where the sensitivity dramatically depends on the power stored in the kilometric cavities. We have shown that thermal problems vanish for the first generation of gravitational antennas if coatings with intrinsic absorption less than $10^{-5}$ can be used; but for the potential next generation of antennas, with a better sensitivity and a correspondingly higher optical power (a gain of one order of magnitude in the sensitivity needs a gain of two orders of magnitude in the stored light power), these thermal effects would be a very important problem ; therefore, it is necessary to start studying ways of compensating for them, both by numerical investigations, as we have begun to do, and by experimental studies. 


\section{Acknowledgements.}

We wish to thank Dr. Nary Man and Dr. Alain Brillet for a number of helpful discussions. We are also grateful to Dr. Peter Fritschel for his careful reading of the manuscript and for his useful comments.

The computation costs have been supported by the Centre National de la Recherche Scientifique, and the calculations have been performed at the CIRCE facility, on the Siemens VP200 machine.

\section{References}

[1] Giazotto A., Interferometric detection of Gravitational Waves, Phys. Rep. 182 (1989) 367.

[2] Forster J. D. and Osterink L. M., Thermal effects in a Nd:YAG Laser, J. Appl. Phys. 41 (1970) 3656.

[3] Apollonov V. V., Barchukov A. I. and Prokhorov A. M., Optical distortion of heated mirrors in $\mathrm{CO}_{2}$-laser systems, IEEE J. Quantum. Electron. QE-10 (1974) 505.

[4] Eggleston J. M., KANe T. J., Kuhn K., Unternahrer J. and Byer R. L., The slab geometry laser. Part I : theory, IEEE J. Quantum. Electron. QE-20 (1984) 289.

[5] FARRUKH U. O., BUONCRISTIANI A. M. and BYVIK C. E., An analysis of the temperature distribution in finite solid-state laser rods, IEEE J. Quantum. Electron. QE-24 (1988) 2253.

[6] InNocenzi M. E., YuRA H. T., Fincher C. L. and FieldS R. A., Thermal modelling of continuouswave end-pumped solid-state lasers, Appl. Phys. Lett. 56 (1990) 1831.

[7] Hello P. and VINET J.-Y., Analytical models of thermal aberrations in massive mirrors heated by high power laser beams, J. Phys. France 51 (1990) 1267.

[8] Hello P. and ViNET J.-Y., Analytical models of transient thermoelastic deformations of mirrors heated by high power cw laser beams, J. Phys. France 51 (1990) 2243.

[9] Cutolo A., Gay P., Solimeno S., Mirror deformations and wavefront aberrations caused by $\mathrm{cw}$ high power laser beams, Opt. Acta 27 (1980) 1105.

[10] The reader must not be surprised by the expression of the steady-state temperature distribution. Indeed, it can be easily shown that the axially-symmetrical steady-state solution of the heat equation can be expressed as

$$
T(r, z)=\sum_{m}\left[A_{m} \cosh \left(k_{m} z\right)+B_{m} \sinh \left(k_{m} z\right)\right] J_{0}\left(k_{m} z\right)
$$

which is a single Dini series (one index). This however can be re-written as a Fourier-Dini series, by expansion of the hyperbolic functions upon the family $\left\{\cos \left(u_{p} z / a\right), \sin \left(v_{p} z / a\right)\right\}$ which is an orthogonal basis for the functions defined within the interval $[-h / 2, h / 2]$.

[11] Hello P., Modèle physique et simulation de l'antenne interférométrique gravitationnelle VIRGO, Thesis (University of Paris XI, 1990).

[12] Hello P. and VINET J.-Y., Modelling of the VIRGO interferometer, in the Proceedings of the Elizabeth and Frederick White Research Conference on Gravitational Astronomy : Instrument Design and Astrophysical Prospects, D. E. McClelland and H.-A. Bachor Eds. (World Scientific, Singapore, 1991) pp. 1-13.

[13] Vinet J.-Y., Hello P., Man C. N. and BRILlet A., A high accuracy method for the simulation of non ideal optical cavities, J. Phys. I France 2 (1992) 1287.

[14] Sziklas E. A. and Siegman A. E., Mode calculations in unstable resonators with flowing saturable gain 2 : Fast Fourier Transform method, Appl. Opt. 14 (1975) 1874.

[15] Rempe G., Thompson R. J., Kimble H. J. and Lalezari R., Measurements of ultralow losses in an optical interferometer, Opt. Lett. 17 (1992) 363.

[16] Handbook of mathematical functions, M. Abramowitz and I. A. Stegun Eds. (Dover Publications, Inc., New York, 1972) p. 485.

[17] Handbuch der Physik, Encyclopedia of Physics: Mechanics of Solids II, C. Truesdell Ed. (Springer-Verlag, Berlin, 1972). 